\title{
Relationship Between Parathyroid Hormone Levels and Abdominal Aortic Calcification in Incident Hemodialysis Patients Not Treated with Calcium or Vitamin D
}

Jin He ( $\sim$ hejin19811118@sina.com )

The Chonggang general hospital

Shubei Chen

The Chonggang general hospital

Kewen Mao

The Chonggang general hospital

Xiaoyan Sun

The Chonggang general hospital

Rongjian Nie

The Chonggang general hospital

\section{Research Article}

Keywords: Chronic kidney disease (CKD), Vascular calcification (VC), abdominal aortic calcification (AAC), parathyroid hormone (PTH)

Posted Date: October 27th, 2021

DOI: https://doi.org/10.21203/rs.3.rs-962363/v1

License: (c) (i) This work is licensed under a Creative Commons Attribution 4.0 International License.

Read Full License 


\section{Abstract}

Vascular calcification (VC) and secondary hyperparathyroidism (SHPT) are important causes of high incidence of cardiovascular events in chronic kidney disease (CKD) patients. The relationship between parathyroid hormone (PTH) and VC is very complex. Different studies have inconsistent reports on the effect of PTH on VC. The present study investigated the correlation between PTH levels and abdominal aortic calcification (AAC) in incident hemodialysis patients who did not receive calcium, calciumcontaining phosphorus binders, calcitriol or vitamin D analogs. Our data confirm that serum PTH levels is significantly negatively correlated with AAC within a certain concentration range in incident hemodialysis patients who not treated with calcium or vitamin $\mathrm{D}$.

\section{Introduction}

The incidence and mortality of cardiovascular disease (CVD) significantly increased in chronic kidney disease (CKD) patients. Even after stratification by age, gender, race, and presence of diabetes, CVD mortality in end-stage renal disease (ESRD) patients treated by hemodialysis or peritoneal dialysis is 10 to 20 times higher than in the general population ${ }^{1}$.

Vascular calcification (VC), defined as the inappropriate and pathological deposition of mineral in the form of calcium phosphate salts into the vascular tissues, is a very common complication in CKD patients and associated with significantly increased all-cause and cardiovascular mortality ${ }^{2-5}$. Secondary hyperparathyroidism (SHPT) is another common complication of CKD and has also been associated with increased cardiovascular mortality and CKD progression, especially in CKD stage 3-5 patients $^{6-8}$.

The relationship between VC and parathyroid hormone (PTH) is very complex. A study of 1095 hemodialysis patients (aged 65-88) showed that abdominal aortic calcification (AAC) was more severe in male patients with serum PTH levels within the upper normal range than patients with serum PTH levels within the lower normal range ${ }^{9}$. Another study revealed that in non-dialysis CKD stage 2-5 patients with AAC score $>6$ or pelvic arterial calcifcation (PAC) score $>1$ had higher serum $\mathrm{PTH}^{10}$. In addition, among patients receiving hemodialysis, serum PTH levels were significantly associated with AAC progression ${ }^{11}$. However, in clinical practice, it is very common that PTH levels do not match the severity of AAC. The present study examined the relationship between PTH levels and AAC in incident hemodialysis patients who did not receive calcium, calcium-containing phosphorus binders, calcitriol or vitamin $D$ analogs in order to determine whether PTH levels are associated with the severity of AAC.

\section{Results}

Clinical characteristics and biochemistry of all patients are shown in Table 1. There were 22 males (73.3\%) and 8 females (26.7\%). The mean age was $55.7 \pm 14.47$ years (23-75 years). The cause of ESRD in our patients was diabetes mellitus in 15, glomerulonephritis in 8, systemic hypertension in 6 and 
autosomal dominant poly-cystic kidney disease in 1. Shapiro-Wilk test showed that iPTH, AAC, diastolic blood pressure (DBP), C-reactive protein (CRP), albumin, triglycerides are skewed distribution, and age, serum calcium, serum phosphorus, systolic pressure (SBP), hemoglobin $(\mathrm{Hb})$, serum total protein (TP), alkaline phosphatase (ALP), serum total cholesterol (TC), high-density lipoprotein (HDL) and lowdensity lipoprotein (LDL) are normal distribution. Because AAC is a skewd distribution which Pearson's correlation or multiple linear regression analysis is not suitable, so Spearman's rank correlation analysis was employed to analyze the relationship between AAC and other variables. 
Table 1

Clinical characteristics and biochemistry of whole cohort $(\mathrm{N}=30)$

\section{Age (23-75 years)}

male

Causes of ESRD

diabetes mellitus

glomerulonephritis

systemic hypertension

autosomal dominant poly-cystic

kidney disease

SBP $(116-196 \mathrm{mmHg})$

DBP (61-108 mmHg)

$\operatorname{ACC}(0-14)$

iPTH (31.3-594.5 pg/mL)

serum calcium (0.88-2.27 $\mathrm{mmol} / \mathrm{L})$

serum phosphorus $(0.86-4.15 \mathrm{mmol} / \mathrm{L})$

$\mathrm{Hb}(39-105 \mathrm{~g} / \mathrm{L})$

CRP (1-172 mg/L)

serum total protein $(13.9-80.8 \mathrm{~g} / \mathrm{L})$

Albumin $(21.9-48.9 \mathrm{~g} / \mathrm{L})$

$\operatorname{ALP}(49-154 \mathrm{U} / \mathrm{L})$

Triglycerides $(0.79-3.89 \mathrm{mmol} / \mathrm{L})$

total cholesterol $(2.33-6.63 \mathrm{mmol} / \mathrm{L})$

HDL $(0.59-1.89 \mathrm{mmol} / \mathrm{L})$

\section{$2.35 \pm 0.75$}

ESRD,end-stage renal disease; SBP, systolic pressure; DBP, diastolic blood pressure; ACC,abdominal aortic calcification; CRP, C-reactive protein; Hb, hemoglobin; ALP, alkaline phosphatase;HDL, highdensity lipoprotein; LDL, low-density lipoprotein; iPTH, intact parathyroid hormone.

Our results suggested that there was a strong negative correlations between serum PTH and AAC (Spearman's rho $-0.618, P<0.001$ ). There was a strong positive correlations between age and AAC (Spearman's rho 0.605, $P<0.001)$. We also found that there was a moderate negative correlation between $\mathrm{Pi}$ and AAC (Spearman's rho -0.435 P=0.016) (Table 2).
$55.7 \pm 14.47$

$73.3 \%$

$15(30)$

$8(30)$

$6(30)$

1 (30)

$155.17 \pm 21.15$

$84.63 \pm 14.50$

$3.27 \pm 4.29$

$242.95 \pm 145.51$

$1.75 \pm 0.34$

2.12 \pm 0.69

$77.2 \pm 16.41$

$20.63 \pm 35.38$

$62.57 \pm 9.22$

$36.47 \pm 7.23$

$95.2 \pm 33.93$

$1.82 \pm 0.88$

$4.10 \pm 1.04$

$1.11 \pm 0.35$ 
Table 2

Spearman's rank correlation analysis for relationship between abdominal aortic calcifcation scores and baseline characteristics.

\begin{tabular}{|c|c|c|}
\hline & $\begin{array}{l}\text { Spearman's rho } \\
\text { AAC }\end{array}$ & P-value \\
\hline Age & 0.605 & $0.000^{* *}$ \\
\hline iPTH & -0.618 & $0.000 * \star$ \\
\hline serum phosphorus & -0.435 & $0.016^{*}$ \\
\hline serum calcium & 0.159 & 0.401 \\
\hline SBP & -0.136 & 0.475 \\
\hline DBP & -0.268 & 0.153 \\
\hline $\mathrm{Hb}$ & 0.283 & 0.130 \\
\hline CRP & -0.081 & 0.669 \\
\hline serum total protein & -0.062 & 0.746 \\
\hline albumin & 0.067 & 0.725 \\
\hline ALP & 0.086 & 0.653 \\
\hline triglycerides & 0.050 & 0.794 \\
\hline total cholesterol & 0.300 & 0.108 \\
\hline HDL & 0.245 & 0.192 \\
\hline LDL & 0.210 & 0.265 \\
\hline
\end{tabular}

SBP, systolic pressure; DBP, diastolic blood pressure; ACC,abdominal aortic calcification; CRP, C-reactive protein; $\mathrm{Hb}$, hemoglobin; ALP, alkaline phosphatase; $\mathrm{HDL}$, high-density lipoprotein; LDL, low-density lipoprotein; iPTH, intact parathyroid hormone.

*: correlation is significant at the 0.05 level; **: correlation is significant at the 0.01 level.

To further analyze why there is a negative correlation between serum phosphorus and AAC, the patients were divided into two groups based on the cut-off point of 50 years old. The AAC and serum PTH of the two groups were skewed distribution, the calcium and phosphorus was normal distribution, so we used Mann-Whitney $U$ test and T-test to compare between groups respectively. 
Our results showed that the serum phosphorus and serum PTH of patients under 50 years old $(10 / 30)$ was significantly higher $(2.62 \mathrm{mmol} / \mathrm{L}$ vs $1.88 \mathrm{mmol} / \mathrm{L}, T$-test, $P=0.004 ; 348.47 \mathrm{pg} / \mathrm{ml}$ vs $190.19 \mathrm{pg} / \mathrm{ml}$, Mann-Whitney $U$ test, $P=0.002)$ than that of patients over 50 years old $(20 / 30)$, and the AAC score was significantly lower than that in patients over 50 years old (0.100 vs 4.85, Mann-Whitney Utest, $P \otimes 0.001$ ). There was no significant difference in serum calcium between the two groups $(1.63 \mathrm{mmol} / \mathrm{L}$ vs 1.80 $\mathrm{mmol} / \mathrm{L} \otimes T$-test, $P=0.315$ ) (Table 3).

Table 3. Groups analysis between different age groups ( $<50$ years old or $\geq 50$ years old).

\begin{tabular}{|c|c|c|c|}
\hline & $\begin{array}{l}\text { Group } 1(<50) \\
\mathrm{N}=10\end{array}$ & $\begin{array}{l}\text { Group } 2(\geq 50) \\
N=20\end{array}$ & P-value \\
\hline Age & $39 \pm 8.72$ & $64.05 \pm 7.96$ & \\
\hline AAC & 0.10 & 4.85 & $\otimes 0.001 *$ \\
\hline iPTH & $348.47 \pm 157.19$ & $190.19 \pm 108.74$ & $0.002^{\star}$ \\
\hline serum phosphorus & $2.62 \pm 0.68$ & $1.88 \pm 0.58$ & $0.004^{\star}$ \\
\hline serum calcium & $1.63 \pm 0.49$ & $1.80 \pm 0.22$ & 0.315 \\
\hline
\end{tabular}

ACC,abdominal aortic calcification; iPTH, intact parathyroid hormone.

*: significant $P$-value $<0.01$.

\section{Discussion}

The present study investigated the correlation between PTH levels and AAC in incident hemodialysis patients who did not receive calcium, calcium-containing phosphorus binders, calcitriol or vitamin D analogs. Our results suggested that lower serum PTH levels were associated with higher AAC scores in this population.

Although the prevalence of VC in CKD patients is higher, the incidence of arterial calcification in different sites is not consistent, and the risk factors of arterial calcification in different locations and their influence on cardiovascular events are also different ${ }^{12-14}$.

AAC is an independent risk factor for all-cause mortality or CVD events in the non-CKD patients, peritoneal dialysis patients and hemodialysis patients ${ }^{15-17}$. The Kidney Disease Improving Global Outcomes (KDIGO) guidelines also suggest that a lateral abdominal radiograph can be used to detect the presence or absence of vascular calcification in patients with CKD stage 3-5 to guide the management of chronic kidney disease-mineral and bone disorder (CKD-MBD) ${ }^{18}$. 
The researches have demonstrated that PTH receptors exist in myocardial cells, vascular smooth muscle cells and endothelial cells, indicating that inappropriate (excessive or insufficient) secretion of PTH may have adverse effects on the cardiovascular system ${ }^{19,20}$. It has been found that PTH perfusion can lead to intense aortic medial calcification in rats with parathyroidectomy and this effect has nothing to do with uremia or serum phosphorus levels ${ }^{21}$. Another study also found that cinacalcet could inhibit the calcification of aorta and heart in 5/6 nephrectomized rats by by decreasing serum PTH levels ${ }^{22}$. These results suggest that PTH has a direct pro-calcification effect, at least in the animal model of CKD.

As for the exact effects that PTH has on AAC in CKD patients, this is still a matter of debate. Studies have shown that serum PTH level is related to the severity of $A A C$ 9, 10,11. However, some studies have also found that there is no association between the two or even negative correlation ${ }^{12-14,23,24}$. One possible explanation for these contradictory conclusions is that the widespread use of calcium, calciumcontaining phosphorus binder, calcitriol or vitamin D analogs affects the natural process of AAC.

Currently, calcium, calcium-containing phosphorus binder, calcitriol or vitamin D analogs are widely used to treat mineral metabolism abnormalities. The DOPPS study found that up to $52 \%$ of participants received vitamin D supplementation; $72.9 \%$ of participants used calcium-containing phosphorus binder for the control of hyperphosphatemia ${ }^{8}$. However, if the above drugs are used improperly, it may lead to adverse clinical consequences. For example, prolonged and disproportionate consumption of vitamin $D$ supplements may lead to excessive inhibition of PTH and aggravation of vascular calcification ${ }^{25}$, 26 ; the use of high-dose calcium salts (oral calcium or calcium-based phosphate binders) can easily lead to hypercalcemia, resulting in low serum PTH levels and vascular calcification ${ }^{27,28}$. Therefore, it is difficult to draw reliable conclusions when discussing the association between PTH and AAC in the CKD population using calcium and vitamin D.

In view of this, in this study, we purposely selected incident hemodialysis patients who have not used calcium, calcium-containing phosphorus binders, calcitriol or vitamin $D$ analogs as the research objects. Interestingly, even after eliminating the interference factors such as calcium and vitamin D, PTH and AAC are still significantly negatively correlated. For the negative correlation, one explanation is that lower serum PTH levels may lead to adynamic bone disease, and adynamic bone disease will impair the ability of patients handling and buffering of calcium loads and pose a higher risk of extraosseous calcifications 29, 30. It should be emphasized that although lower serum PTH levels do contribute to the risk of adynamic bone disease, there is currently no evidence that low PTH alone can represent adynamic bone disease. Another possible explanation for the negative correlation between AAC and PTH is that low serum PTH levels may only be a manifestation of malnutrition, inflammation or cachexia syndrome (MICs) and malnutrition-inflammation is associated with vascular calcification in uremic patients ${ }^{31}$. However, in another study that included 97 hemodialysis patients who were followed up for one year, patients with malnutrition and chronic inflammation (defined as serum albumin $<40 \mathrm{~g} / \mathrm{L}$ and hs$\mathrm{CRP} \geq 28.57 \mathrm{nmol} / \mathrm{L}$ ) had significantly higher PTH levels than the control group ( $241.5 \mathrm{pg} / \mathrm{ml}$ vs 161.8 $\mathrm{pg} / \mathrm{ml})^{11}$. Therefore, neither adynamic bone disease nor malnutrition can fully explain why low PTH 
levels can aggravate AAC. Further studies are needed to elucidate the mechanism of AAC deterioration caused by low PTH levels.

Another surprising finding of the present study is that there is also a significant negative correlation between AAC and serum phosphorus. Serum phosphorus plays a very important role in the occurrence and development of vascular calcification ${ }^{32}$. Subgroup analysis of the MESA study indicated that each $1-\mathrm{mg} / \mathrm{dl}$ increment in serum phosphate concentration was associated with a $21 \%, 33 \%, 25 \%$ and $62 \%$ greater prevalence of coronary artery, thoracic, aortic valve, and mitral valve calcification, respectively ${ }^{33}$. However, some researchers found that there was no significant difference in serum phosphorus levels between the AAC score $>6$ group and AAC score $\leq 6$ group in hemodialysis patients ${ }^{10}$; Study on chinese hemodialysis population (CDCS study) also found that serum phosphorus is a risk factor for coronary artery calcification, but not a risk factor for $A A C{ }^{13}$. It should be pointed out that there are deficiencies in the above studies, that is, all participants received hemodialysis or peritoneal dialysis which can effectively remove serum phosphorus and a large proportion of the participants took phosphate binders (64.6\% in CDCS study). Therefore, it can not concluded that there is no correlation between serum phosphorus and AAC. In the present study, serum phosphorus was not affected by dialysis, and the patient did not use any form of phosphorus bonding agent, but there is still a significant negative correlation between AAC and serum phosphorus. This finding is consistent with that of Harin Rhee et al 31 , who also found that the prevalence of baseline AAC and its progression in low serum phosphorus group was significantly higher than that in high serum phosphorus group.

Further analysis of the patients' characteristics showed that the serum phosphate of patients under 50 years old was significantly higher than that of patients over 50 years old, but the AAC score was significantly lower than that in patients over 50 years old. We speculate that age may have a greater impact on AAC than serum phosphorus in CKD patients. Indeed, a study of young patients with ESRD who were undergoing dialysis also confirmed that there was no significant difference in serum phosphorus between patients with or without coronary artery calcification ${ }^{28}$. Another study involving 174 Chinese patients also found that age may be the most important factor affecting coronary artery calcification in maintenance hemodialysis patients ${ }^{34}$.

In our study, most of the patients were relatively young with an

average age of 55.7 years, so the effect of phosphorus on AAC may not be obvious. Therefore, serum phosphorus may be statistically negatively correlated with AAC, but it does not mean that serum phosphorus has no effect on AAC from a pathophysiological perspective. Our results once again show that the mechanism of $\mathrm{VC}$ is very complex, and even serum phosphorus, a recognized pro-calcification factor, has different effects on specific arteries in specific CKD populations.

There are several limitations to our study. First, the sample size for our study was small. More CKD stage $5 \mathrm{D}$ patients who did not receive calcium, calcium-containing phosphorus binder, calcitriol or vitamin $\mathrm{D}$ analogs would be necessary to attain adequate power in determining the correlation between serum PTH 
levels and AAC. Unfortunately, this was beyond our capacity. Second, We only evaluated the degree of AAC by abdominal radiographs, which is less sensitive and accurate than electron beam computed tomography (EBCT), multislice CT (MSCT). However, due to the relatively high cost and the risk of exposure to higher radiation doses, these tests cannot be performed routinely. Third, the serum PTH levels of our observation population was in the range of $31.3-594.5 \mathrm{pg} / \mathrm{ml}$. The correlation between PTH and AAC is not clear in CKD patients with serum PTH levels exceeding $600 \mathrm{pg} / \mathrm{ml}$ or higher.

In conclusion, the present study, which is the only study focused on the association between PTH levels and $A A C$ in incident hemodialysis patients who did not receive calcium, calcium-containing phosphorus binder, calcitriol or vitamin D analogs, demonstrates that PTH levels is significantly negatively correlated with AAC within a certain concentration range. Inappropriate inhibition of PTH may lead to deterioration of AAC in CKD stage 5D patients.

\section{Methods}

\section{Patients}

This study was approved by the Ethical Committee of the Chonggang general hospital. Written informed consent was obtained from each person at recruitment. We confirm that all experiments of the study were performed in accordance with relevant guidelines and regulations. Incident hemodialysis patients who have not used calcium, calcium-containing phosphorus binders, calcitriol or vitamin D analogsage between January 2020 and june 2021 were initially screened for enrollment in the cross-sectional study. Of the total 47 incident hemodialysis patients, patients with malignancy, decompensated liver Cirrhosi, lupus nephritis, crescentic glomerulonephritis and acute kidney injury (AKI), as well as the elderly over 75 years old were excluded. Finally, 30 patients were enrolled.

\section{Biochemical data and vascular calcifcation}

Pre-dialysis blood samples and plain radiographs were obtained at the time of enrollment. Serum intact parathormone hormone (iPTH) levels were determined by Chongqing DIAN medical laboratory ( reference range $15-65 \mathrm{pg} / \mathrm{ml}$ ). AAC was determined by a lateral lumbar spine radiograph, and quantitative analysis of AAC was performed using the method previously described by kauppila et al. (total score $0-24)^{35}$. All Xrays were reviewed by two physicians who had the expertise to score the X-rays.

\section{Statistical analysis}

Data are presented as mean \pm standard deviation (SD). The normality of the distribution was determined using the Shapiro-Wilk test. The correlation between two continuous variables was analyzed by the Pearson's correlation (normal distribution) or Spearman's correction (Skew distribution). Two continuous variables were compared using the Student's t test (normal distribution) or non-parametric test (Skew distribution). $P$ value $<0.05$ is considered statistically signifcant. All computations were performed using the SPSS 20.0 software (Chicago, IL, USA). 


\section{Declarations}

Acknowledgements.

Not applicable.

\section{Authors' contributions}

jin he conceived and wrote the manuscript. jin he, Shubei Chen ,Kewen Mao, Xiaoyan Sun collected clinical date. jin he and Rongjian Nie reviewed lateral lumbar spine radiograph and performed semiquantitative scoring. jin he, Shubei Chen, Kewen Mao, Xiaoyan Sun and Rongjian Nie confirm the authenticity of all the raw data. All authors have read and approved the final manuscript.

\section{Additional Information}

\section{Conflict of interest.}

The authors declare that they have no competing interests.

\section{References}

1. Foley, R. N., Parfrey, P. S. \& Sarnak, M. J. Clinical epidemiology of cardiovascular disease in chronic renal disease.Am J Kidney Dis. 1998Nov;32(5 Suppl 3):S112-9.

2. Neil, J., Paloian, Cecilia, M. \& Giachelli A current understanding of vascular calcification in CKD.Am J Physiol Renal Physiol. 2014 Oct15; 307(8):F891-F900.

3. Masahide Mizobuchi, D., Towler, E. \& Slatopolsky Vascular calcification: the killer of patients with chronic kidney disease. J Am Soc Nephrol, 20 (7), 1453-1464 (2009 Jul).

4. Russo, D. et al. Progression of coronary artery calcification and cardiac events in patients with chronic renal disease not receiving dialysis.Kidney. 2011 Int80:112-118.

5. London, G. M. et al. Arterial media calcification in end-stage renal disease: impact on all-cause and cardiovascular mortality. Nephrol Dial Transplant, 18, 1731-1740 (2003).

6. Milica Bozic, J. M. et al. Independent effects of secondary hyperparathyroidism and hyperphosphatemia on chronic kidney disease progression and cardiovascular events: an analysis from the NEFRONA cohort. Nephrol Dial Transplant. 2021 May 21;gfab184.

7. Glen, T. et al. Impact of secondary hyperparathyroidism on disease progression, healthcare resource utilization and costs in pre-dialysis CKD patients. Curr Med Res Opin, 24 (11), 3037-3048 (2008 Nov).

8. Francesca Tentori, M. J. et al. Mortality risk for dialysis patients with different levels of serum calcium, phosphorus, and PTH: the Dialysis Outcomes and Practice Patterns Study (DOPPS).Am J Kidney Dis. 2008Sep;52(3):519-30. 
9. Buizert, P. J., van Schoor, N. M. \& Simsek, S. PTH: A New Target in Arteriosclerosis? J Clin Endocrinol Metab2013 Oct;98(10)

10. Sinee Disthabanchong, K. et al. Abdominal aorta and pelvic artery calcifcations on plain radiographs may predict mortality in chronic kidney disease, hemodialysis and renal transplantation.Int Urol Nephrol2018 Feb;50(2)

11. Choi, S. R. et al. Malnutrition, inflammation, progression of vascular calcification and survival: Interrelationships in hemodialysis patients. PLoS One. 2019 May 2;14(5):e0216415.

12. Qingyu Niu, H. \& Zhao, B. Wu. Study on the Prevalence of Vascular Calcification in Different Types of Arteries and Influencing Factors in Maintenance Peritoneal Dialysis Patients. Blood Purif, 47 (Suppl 1(Suppl 1), 8-16 (2019).

13. Liu, Z. H. \& Yu, X. Q. Jun-Wei. Prevalence and risk factors for vascular calcification in Chinese patients receiving dialysis: baseline results from a prospective cohort study.Curr Med Res Opin 2018Aug; 34(8):1491-1500.

14. Qingyu \& Niu Huiping Zhao,Bei Wu. Abdominal aortic calcification is superior to other arteries calcification in predicting the mortality in peritoneal dialysis patients - a 8 years cohort study. BMC Nephrol, 20, 439 (2019).

15. Reza Golestani, R. Ã. et al. Abdominal aortic calcification detected by dual X-ray absorptiometry: A strong predictor for cardiovascular events. Ann Med, 42 (7), 539-545 (2010 Oct).

16. Francesca Martino, P. D. et al. Abdominal aortic calcification is an independent predictor of cardiovascular events in peritoneal dialysis patients. Ther Apher Dial, 17 (4), 448-453 (2013 Aug).

17. Xuying Zhu, H. et al. Association of abdominal aortic calcification estimated by plain radiography with outcomes in hemodialysis patients: a six-year follow-up study. Nephrology (Carlton), 25 (7), 559-565 (2020 Jul).

18. Kidney Disease: Improving Global Outcomes (KDIGO) CKD-MBD Update Work Group. KDIGO 2017 Clinical Practice Guideline Update for the Diagnosis, Evaluation, Prevention, and Treatment of Chronic Kidney Disease-Mineral and Bone Disorder (CKD-MBD). Kidney Int Suppl, 7, 1-59 (2017).

19. Tomaschitz, A. et al. Aldosterone and parathyroid hormone interactions as mediators of metabolic and cardiovascular disease.Metabolism. 2014Jan;63(1):20-31.

20. Chen, H. et al. Parathyroid Hormone Fragments: New Targets for the Diagnosis and Treatment of Chronic Kidney Disease-Mineral and Bone Disorder.Biomed Res Int. 2018 Nov29;2018:9619253.

21. Neves, K. R. et al. Vascular calcification: contribution of parathyroid hormone in renal failure. Kidney Int, 71 (12), 1262-1270 (2007 Jun).

22. Takehisa Kawata, N. et al. Cinacalcet suppresses calcification of the aorta and heart in uremic rats.Kidney Int. 2008Nov;74(10):1270-7.

23. Ahmed Fayed, Mahmoud, M. et al. Calcification of abdominal aorta in patients recently starting hemodialysis: A single-center experience from Egypt. Saudi J Kidney Dis Transpl. Jul-Aug, 30 (4), 819-824 (2019). 
24. Sul, A. et al. Low serum intact parathyroid hormone level is an independent risk factor for overall mortality and major adverse cardiac and cerebrovascular events in incident dialysis patients. Osteoporos Int, 27 (9), 2717-2726 (2016 Sep).

25. M Shawkat Razzaque. The dualistic role of vitamin $D$ in vascular calcifications. Kidney Int, 79 (7), 708-714 (2011 Apr).

26. Masahide Mizobuchi, H. et al. Vitamin D and vascular calcification in chronic kidney disease., 45 (Suppl 1), S26-9 (2009 Jul).

27. Glenn, M. et al. Sevelamer attenuates the progression of coronary and aortic calcification in hemodialysis patients. Kidney Int, 62 (1), 245-252 (2002 Jul).

28. Goodman, W. G., Goldin, J. \& Kuizon, B. D. Coronary-artery calcification in young adults with endstage renal disease who are undergoing dialysis. N Engl J Med, 18 (20), 1478-1483 (2000 May).

29. Kurz, P. et al. Evidence for abnormal calcium homeostasis in patients with adynamic bone disease. Kidney Int, 46 (3), 855-861 https://doi.org/10.1038/ki.1994.342 (1994 Sep).

30. Cannata, J. B. Andí. Adynamic bone and chronic renal failure: an overview. Am J Med Sci, 320 (2), 81-84 (2000 Aug).

31. Harin Rhee, S. H. et al. Persistently low intact parathyroid hormone levels predict a progression of aortic arch calcification in incident hemodialysis patients. Clin Exp Nephrol, 16 (3), 433-441 (2012 Jun).

32. Navid Shobeiri, M. A., Adams, Rachel, M. \& Holden Phosphate: an old bone molecule but new cardiovascular risk factor. Br J Clin Pharmacol, 77 (1), 39-54 (2014 Jan).

33. Kathryn, L., Adeney, D. S., Siscovick, Joachim, H. \& Ix Association of serum phosphate with vascular and valvular calcification in moderate CKD. J Am Soc Nephrol, 20 (2), 381-387 (2009 Feb).

34. Wen, Y. et al. Safety of Low-calcium Dialysate and its Effects on Coronary Artery Calcification in Patients Undergoing Maintenance Hemodialysis.Sci Rep2018 04 13;8(1).

35. Kauppila, L. I. et al. New indices to classify location, severity and progression of calcific lesions in the abdominal aorta: a 25-year follow-up study. Atherosclerosis, 25 (2), 245-250 (1997 Jul). 